\title{
Mycolactones: immunosuppressive and cytotoxic polyketides produced by aquatic mycobacteria
}

\author{
Hui Hong, ${ }^{a}$ Caroline Demangel, ${ }^{b}$ Sacha J. Pidot, ${ }^{c}$ Peter F. Leadlay ${ }^{a}$ and Tim Stinear ${ }^{* c}$
}

\author{
Received 25th February 2008 \\ First published as an Advance Article on the web 17th April 2008 \\ DOI: 10.1039/b803101k
}

Covering: up to January 2008

\begin{abstract}
Mycolactones are a family of highly related macrocyclic polyketides that exhibit immunosuppressive and cytotoxic properties. First discovered in 1999, they are the primary virulence factors produced by the environmental human pathogen Mycobacterium ulcerans, the causative agent of Buruli ulcer, and by some closely-related aquatic mycobacteria that cause disease in fish and frogs. Mycolactones are characterized by a common 12-membered lactone core to which is appended an unsaturated fatty acyl side-chain of variable length and oxidation state. This Highlight summarizes recent progress in understanding the structural diversity of the mycolactones, their biological activity and mode of action in mammalian cells, and the genetics, evolution, and enzymology of their biosynthesis.
\end{abstract}

\section{Introduction}

In the 1960s, pathologists in Uganda studying Buruli ulcer, an unusual skin disease caused by Mycobacterium ulcerans, noted in histological sections that tissue necrosis and a marked lack of inflammation extended beyond zones containing bacteria, suggesting that $M$. ulcerans produced a diffusible toxin. ${ }^{1,2}$ In support of this idea, culture filtrates of M. ulcerans were later found to be cytotoxic to eukaryotic cells, provoke necrosis following injection in guinea pig skin or mouse footpads, and to have immunosuppressive properties. ${ }^{3-6}$ Finally, the putative toxin was successfully purified from a clinical isolate of $M$. ulcerans and revealed as a polyketide that was named mycolactone. ${ }^{7,8}$ It was originally identified as a mixture of $c i$ s and trans isomers designated mycolactone $\mathrm{A}$ and $\mathrm{B} .^{7,9}$ Since then it has been shown that different strains of $M$. ulcerans and closely related mycobacterial species naturally produce at least five structurally distinct molecules, each of which likely also exists in cis and trans forms. The importance of mycolactones for the survival of $M$. ulcerans is unknown, although a recent study suggests mycolactones are a major constituent of an abundant extracellular matrix produced by the bacterium that may confer an enhanced capacity to colonize certain ecological niches. ${ }^{10}$

\section{Mycolactones: structural characterization and diversity}

Mycolactones A and B were first isolated from M. ulcerans MU1615, a Malaysian strain that makes the same mycolactones as African isolates of $M$. ulcerans. ${ }^{7,8}$ Their overall structures were shown by $2 \mathrm{D}$ NMR experiments to be, respectively, $Z-\Delta^{4^{\prime}, 5^{\prime}}$ and

${ }^{a}$ Department of Biochemistry, University of Cambridge, 80 Tennis Court Road, Cambridge, CB2 1GA, UK

${ }^{b}$ Unité Postulante Pathogénomique Mycobactérienne Intégrée, Institut Pasteur, 25 Rue du Dr Roux, Paris, 75015, France

${ }^{c}$ Department of Microbiology, Monash University, Wellington Road, Clayton, 3800, Australia
$E-\Delta^{4^{\prime}, 5^{\prime}}$ isomers of a 12 -membered macrocyclic polyketide in which a second highly unsaturated polyketide side-chain is appended via an ester linkage ${ }^{9}$ (Fig. 1). The absolute configuration of mycolactone A and B was established soon afterwards by chemical synthesis. ${ }^{11-13}$ Subsequent investigations of mycolactone structure have been hampered by the small (usually microgram) quantities available from laboratory-scale cultivation. However, either by scaling-up to $150 \mathrm{~L}$ scale, ${ }^{14}$ or by use of sensitive mass spectrometry techniques, ${ }^{15,16}$ analysis of culture extracts of a typical strain of M. ulcerans has revealed the presence of minor amounts of additional mycolactones, differing from mycolactones A and B only in the side-chain. In particular, use of $\mathrm{LC}^{-\mathrm{MS}^{n}}$, combining ion trap mass spectrometry (quadruple ion trap or FT-ICR) with multi-stage collisioninduced fragmentation experiments, ${ }^{15}$ has provided detailed structural information. Such analysis of cell extracts of the African strain MUAgy99 revealed that apart from mycolactone $\mathrm{A} / \mathrm{B}$ with $[\mathrm{M}+\mathrm{Na}]^{+}$at $m / z 765$ as the major species, there were present small amounts of other mycolactones with $[\mathrm{M}+\mathrm{Na}]^{+}$at $m / z 763,749,747,745$ and $781 .^{15}$ Analysis of cell extracts of $M$. ulcerans strains from Malaysia and Japan has revealed very similar mycolactone production profiles (H. H., P. F. L. and T. $\mathrm{S}$., unpublished data). $\mathrm{MS}^{n}$ analysis showed that these mycolactone congeners share the same core lactone structure as mycolactone $\mathrm{A} / \mathrm{B}$, with the structural variations being confined to the distal end of the side-chain. The species with $[\mathrm{M}+\mathrm{Na}]^{+}$at $\mathrm{m} / \mathrm{z} 749$ has been identified, for example, as mycolactone $\mathrm{C}$ (Fig. 1) in which the normal late-stage hydroxylation of the sidechain at $\mathrm{C}-12^{\prime}$ has not taken place. ${ }^{15,16}$ The other species can be accounted for if the polyketide synthase governing the side-chain biosynthesis assembles the chain with less than perfect specificity $^{15}$ (see also sections 4 and 5).

Intriguingly, other clinical isolates of $M$. ulcerans show distinctly different patterns of mycolactone production. For example, Australian strains produce almost exclusively mycolactone $\mathrm{C}\left([\mathrm{M}+\mathrm{Na}]^{+} \text {at } \mathrm{m} / z \text { 749) }\right)^{16,44}$ (Fig. 1). The missing hydroxyl group at $\mathrm{C}-12^{\prime}$ of the side-chain compared to 


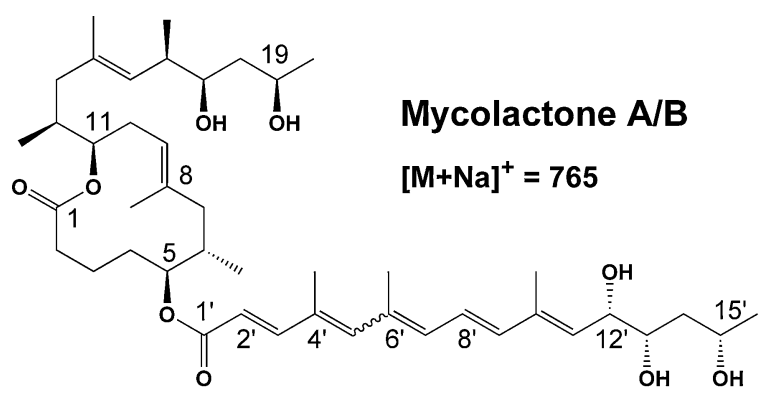

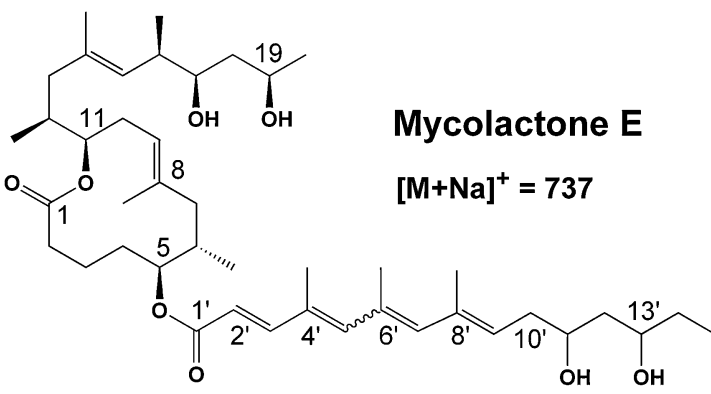

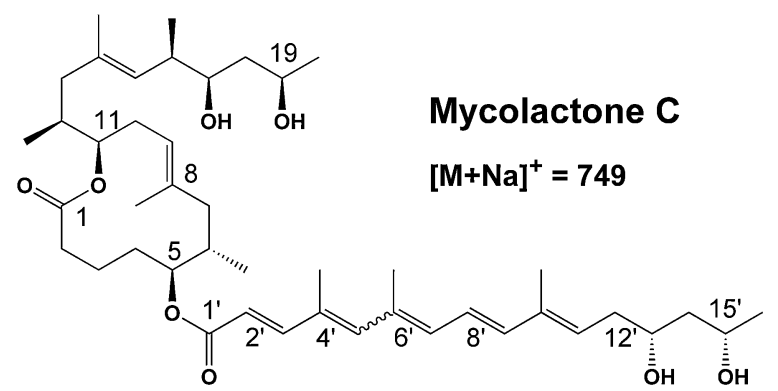<smiles></smiles>

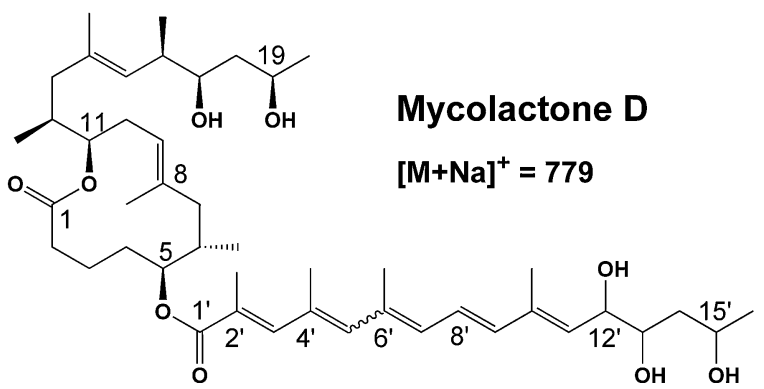

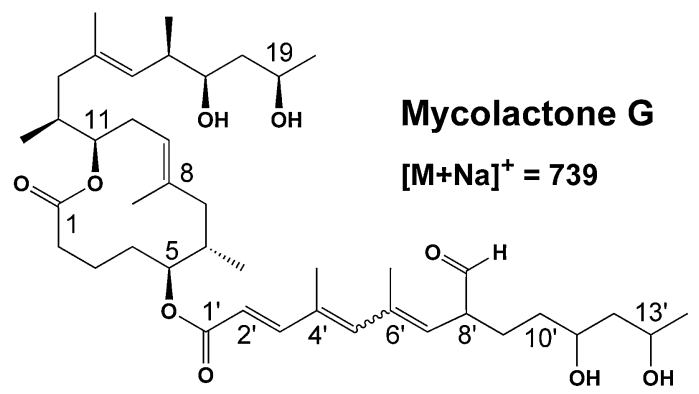

Fig. 1 Mycolactone variations represented by the five naturally occurring structures (A/B-F) and one unnatural structure (G). The stereochemistry of the core is assumed to remain the same but has yet to be established for mycolactones D and E.

mycolactone $\mathrm{A} / \mathrm{B}$ is likely due to the lack in this strain of cyp140A7 (MUP_053), a plasmid-borne gene that encodes a specific P450 mono-oxygenase (see section 5). The structure and the absolute configuration of mycolactone $\mathrm{C}$ have also been confirmed by total synthesis. ${ }^{17}$ Strikingly, the Chinese strain MU98912 produces a new set of mycolactones with $[\mathrm{M}+\mathrm{Na}]^{+}$at $\mathrm{m} / \mathrm{z}$ 779, 777 and 761. Detailed MS analysis pinpointed the distinctive structural difference in this new family as the presence of an extra methyl branch at C-2 ${ }^{\prime}$ of the side-chain. ${ }^{18}$ The new mycolactone with $[\mathrm{M}+\mathrm{Na}]^{+} \mathrm{m} / z 779$ has been named mycolactone D (Fig. 1) (Note that apart from mycolactones A/B, the nomenclature does not make any distinction between geometric (E- and Z-) isomers.) The structural change in mycolactone D correlated perfectly with the finding from DNA sequence

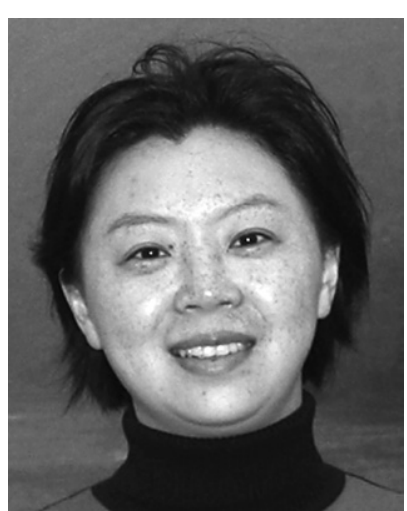

Hui Hong
Hui Hong received her PhD in Chemistry from Nanjing University in 1997. She joined the group of Professors Jim Staunton and Peter Leadlay at Cambridge as a postdoctoral researcher in 2001. Her research interests are the application of mass spectrometry in the study of polyketide natural products and their biosynthesis. She is currently supported by the Wellcome Trust to work on mycolactone.

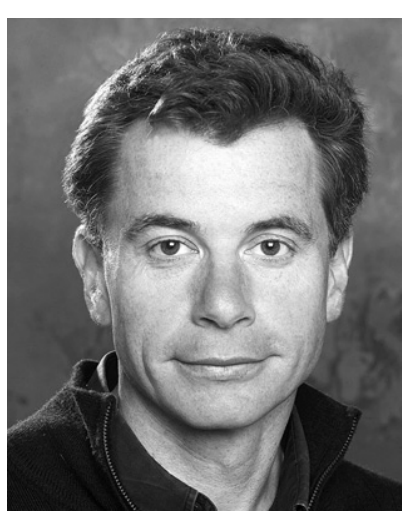

Tim Stinear
Tim Stinear received his $P h D$ in Microbiology from Monash University in 2001 followed by a 3-year postdoc with Professor Stewart Cole at the Institut Pasteur to sequence the genome of Mycobacterium ulcerans. Since 2004 he has led his own research group in the Department of Microbiology at Monash University, studying the genetics and biosynthesis of mycolactones. 
analysis of the corresponding genes in the Chinese strain, that the malonyl-CoA-specific acyltransferase (AT) domain in module 7 of the side-chain polyketide synthase $(\mathrm{PKS})^{19}$ (which governs incorporation of this part of the chain) was substituted by an AT domain specific for methylmalonyl-CoA. ${ }^{18}$ The absolute configuration of mycolactone D remains to be established.

Mycolactones have also been found in aquatic mycobacteria related to M. ulcerans. In 2001, a frog pathogen was discovered and named as Mycobacterium liflandii. ${ }^{20}$ Analysis of the lipid extracts revealed another family of mycolactones, with $[\mathrm{M}+$ $\mathrm{Na}^{+}$at $\mathrm{m} / \mathrm{z} 737$ (major) and 735 (minor). ${ }^{21,22}$ The new mycolactone with $[\mathrm{M}+\mathrm{Na}]^{+}$at $\mathrm{m} / z 737$ was designated as mycolactone E. Alternative structures were initially proposed for mycolactone $\mathrm{E}^{21,22}$ but $\mathrm{MS}$ analysis in combination with deuterium exchange and chemical transformation firmly supports the structure shown in Fig. 1, ${ }^{22}$ in which (compared to mycolactone A/B) the number of conjugated double bonds in the side-chain is reduced from five to four, the terminal C-14' methyl group is replaced by an ethyl group, and there is no hydroxylation at $\mathrm{C}-10^{\prime}$, the position equivalent to $\mathrm{C}-12^{\prime}$ in mycolactone A/B. Mycolactones have also been detected from recently-described fish pathogens, such as Mycobacterium marinum DL045, M. marinum CC240299, $M$. marinum DL240290 and Mycobacterium pseudoshottsii L15. Cell extracts from each of these strains contain mycolactones with $\mathrm{m} / \mathrm{z}$ at 723 (major component) and $m / z 721$ (minor component). ${ }^{23,24}$ The mycolactone with $\mathrm{m} / \mathrm{z} 723$ has been named mycolactone $\mathrm{F}$ (Fig. 1). $\mathrm{MS}^{23}$ and $\mathrm{NMR}^{24}$ analysis have both shown that in terms of overall structure mycolactone $\mathrm{F}$ differs from mycolactone $\mathrm{E}$ only in its lack of the terminal $\mathrm{C}-15^{\prime}$ methyl group in the side-chain. Intriguingly, a recent total synthesis of mycolactone $\mathrm{F}$ by the Kishi group ${ }^{25}$ has defined the absolute configuration of natural mycolactone $\mathrm{F}$ as that shown in Fig. 1, in which the configuration of the $C^{\prime}-11$ and $C^{\prime}-13$ hydroxyl groups in the distal part of the side-chain is reversed from that of the other mycolactones $(\mathrm{A} / \mathrm{B}, \mathrm{C})$ whose absolute configuration is known. The relevant ketoreductase activities of the side-chain PKS (section 5) are predicted to have reversed stereospecificities in such strains, compared to M. ulcerans. It would appear that the structural variation in natural mycolactones extends also to differences in configuration at comparable stereocentres.

\section{Assessment of biological activity and exploration of the mode of action}

Although the precise molecular mechanisms of mycolactone action on eukaryotic cells remain unknown, significant progress has been made in the characterisation of its biological activity, as discussed below (Fig. 2).

\subsection{Uptake and sub-cellular localization}

A derivative of mycolactone bearing a fluorescent reporter group in the side-chain (with 10-fold lower biological activity) has been shown to accumulate in the cytosol of murine fibroblasts in vitro, in a non-saturable and non-competitive manner. ${ }^{26}$ Similar results have been obtained with human epithelial cells and lymphocytes exposed to a ${ }^{14} \mathrm{C}$-labelled form of the toxin, showing that mycolactone accumulates in a time- and dose-dependent fashion in the cell cytoplasm, and not in either the plasma cell membrane

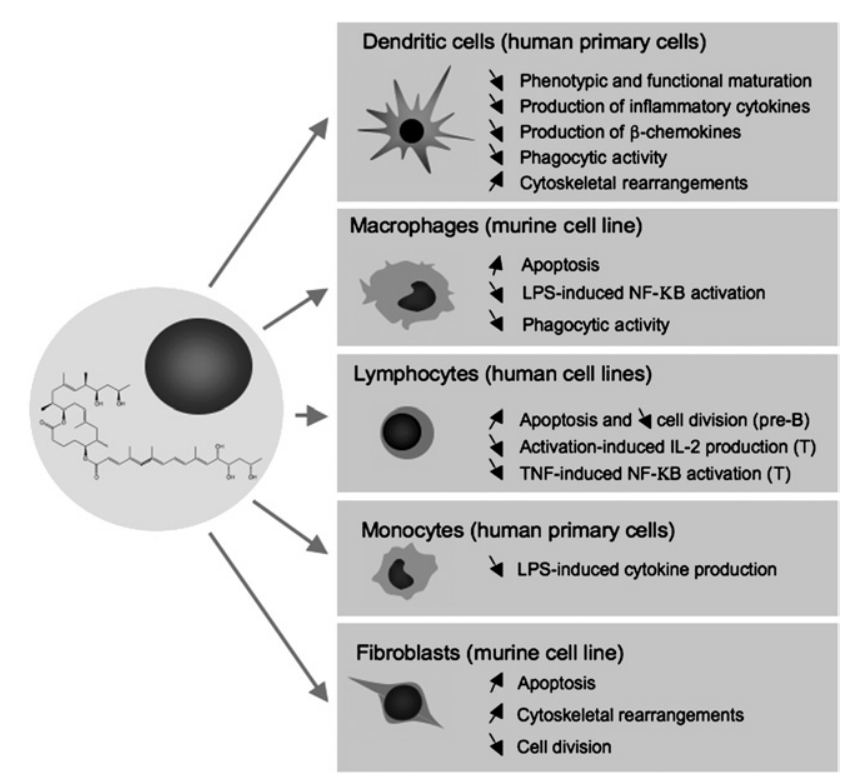

Fig. 2 Summary of the known effects of mycolactones on various mammalian cell types. Abbreviations LPS: lipopolysaccharide; TNF: tumour necrosis factor; IL: interleukin; NF-кB: nuclear factor kappa B.

or the nucleus (C. D., unpublished data). These data are consistent with a non-cell-specific passive diffusion of the toxin through the plasma membrane, followed by interaction with a cytosolic target. Identifying the molecular target(s) and pathway of action of mycolactone would undoubtedly assist the identification of molecules capable of blocking the activity of the toxin in vivo, which would obviously be of therapeutic interest.

\subsection{Cytotoxic effects of mycolactone}

Treatment of murine L929 fibroblasts with mycolactone in vitro triggers, as an early event, the loss of attachment to the extracellular matrix in a dose-dependent manner. Induction of apoptosis, with increases in intracellular calcium and induction of caspases, ${ }^{26}$ arrest in the G0/G1 phase of the cell cycle, and eventual cell death are seen only after 2-3 days of incubation with the toxin, whereas cells become detached and round up after only 24 h of treatment. ${ }^{8}$ In fact, mycolactone causes dramatic cell skeletal rearrangements in L929 cells after only $4 \mathrm{~h}$ of exposure to mycolactone. Such cells show foci of F-actin, and the normal actin stress fibre structures have disappeared. This deleterious effect on the actin skeleton may account for the decreased phagocytic activity of mycolactone-treated macrophages. ${ }^{27-29}$ However, the toxin appears not to interact directly with the actin skeleton, since actin staining never co-localises with a fluorescent derivative of mycolactone in toxin-treated L929 cells ${ }^{26}$ and the in vitro kinetics of actin polymerization and depolymerization are both unchanged in the presence of up to $20 \mu \mathrm{M}$ mycolactone (C. D., unpublished data).

Although the histopathological examination of ulcers suggests that mycolactone activity is not cell-specific, in vitro studies have revealed differences in cellular sensitivity to mycolactone. For example, the toxin blocked the proliferation of a murine pre-B cell line but not a human $\mathrm{T}$ cell line. ${ }^{6}$ Also, the cytotoxicity of mycolactone towards human dendritic cells was higher during 
the immature state. ${ }^{26}$ These observations suggest that the molecular target of mycolactone is ubiquitous, but may be differentially expressed depending on the cell type or the state of activation of the cell.

\subsection{Immunosuppressive effects}

Before mycolactone was purified, the inhibitory action of a $M$. ulcerans lipid extracts on the production of TNF by human monocytes and of TNF-induced NF- $\kappa \mathrm{B}$ activation was noted. ${ }^{6}$ Suppression of TNF production without loss of cell viability was later confirmed by exogenously added, or endogenously produced, toxin in murine macrophages. ${ }^{29,30}$ The suppressive effect of mycolactone on inflammatory cytokines such as TNF was nevertheless marginal in human dendritic cells. However, in these cells, which are considered the key initiators and regulators of immune responses, functional maturation and production of chemokines was abolished in the presence of nanomolar concentrations of mycolactone. ${ }^{31}$ As these chemotactic signals are critical for the induction of local inflammatory responses, this effect of mycolactone may account for the lack of inflammatory infiltrates in infected tissues. ${ }^{31}$ The observations that systemic IFN- $\gamma$ responses are suppressed in Buruli ulcer patients ${ }^{32-38}$ strongly suggest that mycolactone may also affect the biology of $\mathrm{T}$ cells. Further evidence for this comes from the fact that partially purified toxin has been found to inhibit the production of IL-2 by human T cell lines.

\subsection{Structure-function studies}

The structural heterogeneity of mycolactones produced by $M$. ulcerans clinical isolates and other mycolactone-producing mycobacteria raises the question of which structural features determine the cytotoxic and immunosuppressive activities. The absence of the fatty acyl side-chain, for example, renders the macrolactone core $10^{4}$-fold less cytotoxic than mycolactone A/B. ${ }^{16}$ Furthermore, variations in the side-chain strongly influence biological activity. Acetylation of hydroxyl groups, or hydrogenation resulting in saturation of the double bonds, reportedly abrogated the cytotoxicity of mycolactone A/B. ${ }^{7} \mathrm{~A}$ periodate-oxidized mycolactone lacking the hydrophilic end of the side chain showed a 10 -fold reduced activity. ${ }^{26}$ When mycolactone variants were compared in assays of cytokine production by human lymphocytes, mycolactones A/B proved to be the most potent inhibitor of IL-2 production. ${ }^{23}$ Mycolactones C, E and F showed lower inhibitory effects, while an engineered form bearing an aldehyde group in place of the methyl substituent at position $\mathrm{C}-8^{\prime}$ of mycolactone $\mathrm{F}$ (mycolactone $\mathrm{G}$ ) was the least active of all. ${ }^{23}$ Together, these data suggest that the presence of a hydroxyl group on $\mathrm{C}-12^{\prime}$ is critically important for immunosuppression by mycolactone $\mathrm{A} / \mathrm{B}$.

\section{Genetics and evolution of mycolactone biosynthesis}

\subsection{Discovery of the mycolactone biosynthesis PKS gene cluster on a megaplasmid}

Genome sequencing of Mycobacterium ulcerans strain Agy99, a mycolactone A/B-producer, revealed a $174 \mathrm{~kb}$ megaplasmid, named pMUM001. The plasmid harbours three very large genes
( $m l s A 151 \mathrm{~kb}$; $m l s A 27 \mathrm{~kb}$; $m l s B 42 \mathrm{~kb}$ ) encoding type 1 modular polyketide synthases that account for $60 \%$ of the total plasmid sequence. The predicted Mls proteins conform to the conventional assembly-line concept for type I polyketide synthases, ${ }^{39}$ involving the processive decarboxylative condensation of activated thioesters of malonyl- and methylmalonyl-CoA. The observed module and domain structure corresponds to expectations based on mycolactone structure whereby MlsA1 and MlsA2 produce the 12-membered lactone core and MlsB produces the unsaturated side-chain (Fig. 3). Transposon mutagenesis of the $m l s$ genes has confirmed their respective roles in mycolactone synthesis. ${ }^{19}$

\subsection{Mycolactones are produced by a family of closely related mycobacteria}

Despite being assigned various species names such as Mycobacterium ulcerans, Mycobacterium liflandii, and Mycobacterium pseudoshottsii, mycolactone-producing mycobacteria are genetically very closely related and have all evolved from a common Mycobacterium marinum ancestor that acquired the pMUM plasmid. ${ }^{40} M$. marinum without pMUM is an environmental bacterium that is a pathogen of fish, frogs and other ectotherms. Interestingly, while $M$. ulcerans is a major human pathogen, other species of mycolactone-producing mycobacteria have never been isolated from humans and are usually (but not always) associated with disease in fish and frogs. ${ }^{21,41,42}$

\subsection{High DNA sequence repetition among domains of the $m l s$ locus}

One of the most surprising features of the mycolactone PKS is the extraordinary level of sequence identity among domains of the same function. Modular PKS domains routinely share 30$80 \%$ aa identity. For example, intra-module amino acid (aa) identity among the $14 \mathrm{KS}$ domains of the rapamycin cluster is $66 \%{ }^{43}$ In comparison, the 16 modules of the Mls locus have an intra-KS domain aa identity of $97 \%$ (Fig. 3). ${ }^{19}$ Identity scores are even higher among the other Mls domains, ranging from 98.7$100 \%$. The repetitive nature of this locus is highlighted in Fig. 3 where the identical colours for domains or modules indicates both identical function and amino acid sequence (97-100\%). The entire $100 \mathrm{~kb}$ locus, encompassing $\mathrm{mls} A 1, \mathrm{mls} A 2$ and $m l s B$, comprises only $9.5 \mathrm{~kb}$ of unique DNA. ${ }^{19}$ One conclusion from these data is that the region has likely evolved very recently from a series of in-frame recombination and duplication events.

\subsection{The $m l s$ locus is unstable yet stable}

Not surprisingly, the extreme sequence identity between modules leads to instability within the $m l s$ locus. Loss of mycolactone production caused by spontaneous deletion of fragments of the $\mathrm{mls}$ genes is frequently observed in subcultured M. ulcerans. ${ }^{44}$ However, strains isolated from African and Asian countries over a 40-year period all produce the same mycolactone $\mathrm{A} / \mathrm{B}$, suggesting that there are very strong selective forces acting on $M$. ulcerans populations to preserve those bacteria that produce mycolactones. The vital role that mycolactones appear to play for M. ulcerans remains to be discovered. 


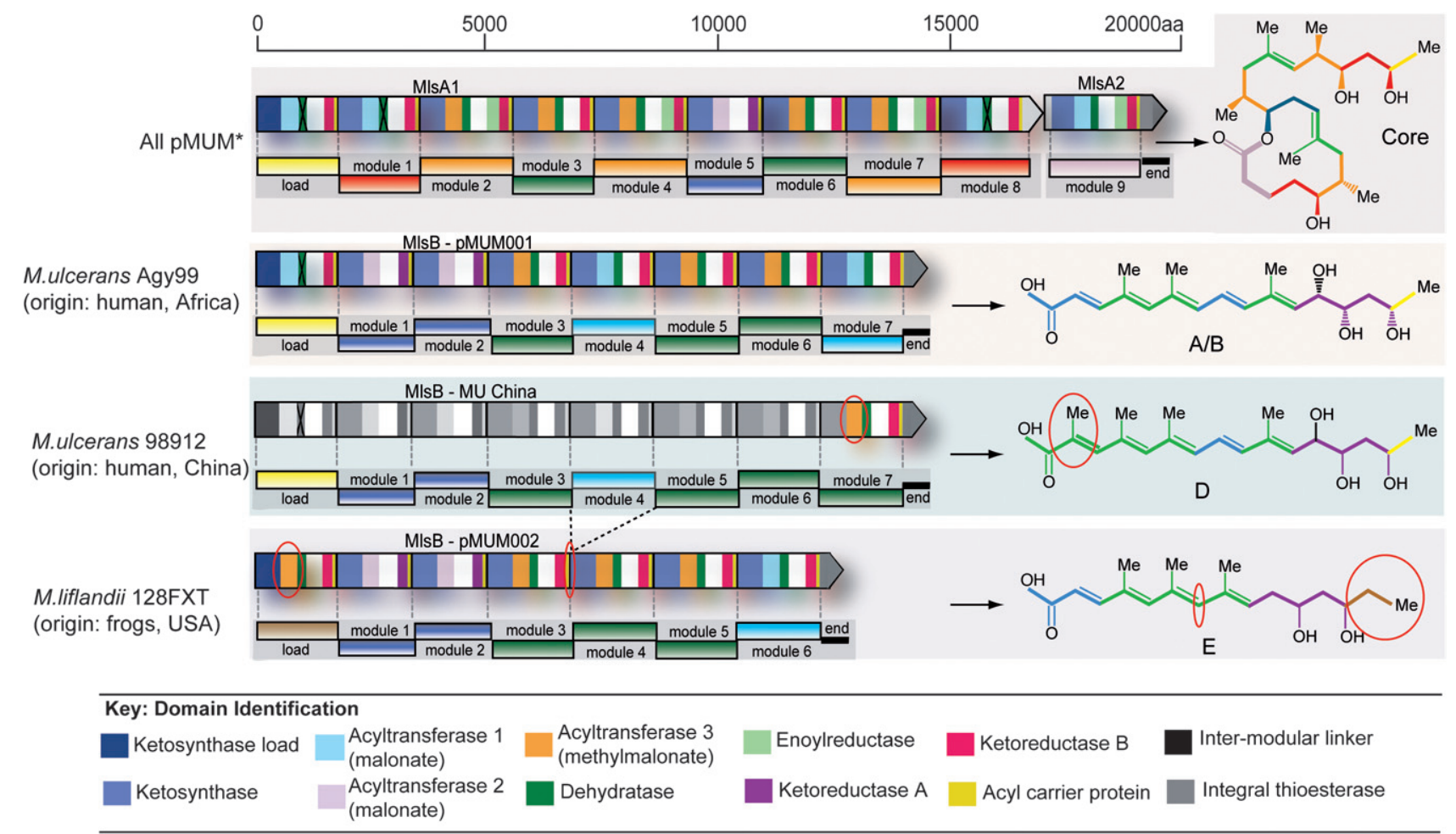

Fig. 3 Genetic organisation of the mycolactone biosynthetic PKS gene loci derived from the complete sequences of pMUM001 (from a mycolactone A/B producer, M. ulcerans Agy99) and pMUM002 (from the mycolactone E producer, Mycobacterium liflandii 128FXT) and proposed from the structure of mycolactone D and the partial sequence of the $m l s B$ gene of $M$. ulcerans strain 980912 (region sequenced shown in colour). The predicted enzymatic domain specificity derived from each of the three proteins (MlsA1, MlsA2 and MlsB) is represented by a coloured block whose predicted function is outlined in the key. Module arrangements are shown below each gene, and modules are colour-coded to reflect the combination of domains they possess. Crosses through dehydratase domains indicate that they are predicted to be inactive based on a mutation in the active site sequence. All identically coloured domains share $97-100 \%$ aa sequence identity.

\subsection{Mycolactone structural variants arise from natural domain swapping and module deletion}

The ongoing domain and module replacement among mycolactone-producing mycobacteria also promotes mycolactone metabolite diversity. Mycolactone D arises by a naturally occurring domain swap in an M. ulcerans isolate from China. In this strain there has been an exchange of the AT domain in module 7 of MlsB from acetate to propionate specificity, leading to an additional methyl branch at C-2' of the mycolactone D side chain (Fig. 1) ${ }^{18}$ Very recently, complete sequence analysis of the $\mathrm{mls}$ locus from Mycobacterium liflandii, a mycolactone E producer, ${ }^{21}$ has shown that the structure of this mycolactone can be explained by MlsB modifications that include replacement of the AT domain of the loading module (acetate to propionate) and precise deletion of an entire extension module (Fig. 3; S. P., H. H., P. F. L. and T. S., manuscript in preparation).

\section{Enzymology of PKS and accessory enzymes}

At first sight, the arrangement of the modules of the mycolactone PKS and of their constituent domains (Fig. 3) provides a compelling vindication of the general modular paradigm for polyketide synthases, with a satisfyingly close correlation between the order and identity of the enzyme activities inferred from the sequencing of the $m l s A$ and $m l s B$ genes of $M$. ulcerans Agy99, and the activities predicted to be required to construct the core and the side-chain of mycolactone A/B. ${ }^{19}$ However, there are certain unusual features of the mycolactone PKS which prompt a closer analysis. First, the sheer size of the PKS multienzymes is unprecedented, with MlsA1 and MlsB housing eight and seven extension modules respectively, in polypeptides containing 16990 aa and 14130 aa respectively. Secondly, the level of mutual sequence identity between comparable domains is wholly unprecedented, and provides a unique opportunity to explore the molecular basis of selectivity and stereoselectivity in polyketide chain extension on modular PKSs. Most strikingly, the ketosynthase (KS) domains in all 16 extension modules have essentially identical amino acid sequences, ${ }^{19}$ which (for this system at least) allows the remarkable conclusion that the overall fidelity of the PKS does not require the KS to discriminate between alternative substrates. It remains to be established whether this outcome is favoured by the arrangement of essentially all the modules of each $m l s$ PKS in a single giant polypeptide, where the correct sequence of modules is guaranteed. The near-identity of KS domains in MlsA and MlsB raises the intriguing question of what in this system prevents premature transfer of polyketide chains to the next module before the correct level of reductive processing has occurred. The observed fidelity of the Mls PKS (especially in synthesis of the core) 
suggests that KS-mediated inter-module transfer is either uniformly slow relative to the rates of reductive steps, or that the $\mathrm{KS}$ has an intrinsic preference for reduced substrates in this transfer reaction. Finally, the $m l s$ gene cluster contains three additional genes proposed to play essential roles in toxin biosynthesis, but where until recently biochemical or genetic evidence for these roles has been lacking.

\subsection{Studies of discrete mycolactone PKS domains in vitro}

As yet, there is no convenient system available for genetic manipulation of $m l s$ PKS genes in M. ulcerans, and there have been no reports of successful heterologous expression of these genes in a faster growing, more genetically tractable host. However, there has been progress in the expression and purification of individual domains in recombinant E. coli, and study of their reaction with surrogate substrates. In particular, a detailed analysis has been published on the ketoreduction of model diketide thioester substrates by individual ketoreductase (KR) domains excised from the mycolactone PKS. ${ }^{45}$ The core lactone PKS MlsA contains an A-type KR domain (KR-A) in extension module 5, and the side-chain PKS MlsB contains two such KR-A domains in extension modules 1 and 2. All the other extension modules contain B-type KR domains (the designations A and B refer to the alternative stereochemistries of reduction which are correlated with specific amino acid motifs in the KR active sites). ${ }^{46}$ Although the KR-B domains are $100 \%$ identical to each other, as are the KR-A domains to each other, both types of KR process PKS-bound polyketide intermediates of several different lengths and incorporating different structural features. It was found that, while the KR-A domain acted on a diketide substrate with complete stereospecificity and stereoselectivity, the KR-B domain showed low stereochemical fidelity. Taken together, these results underline the small energetic differences between alternative modes of presentation of the substrates to the KR active site, and the likely importance of the tethering of polyketide intermediates to ACP domains in ensuring the correct stereochemical outcome. $^{45}$

Another intriguing feature of the mycolactone PKS is presented by the C-terminal domains of MlsA2 and MlsB, which are each predicted to house a thioesterase/acyltransferase/cyclase (type I TE) activity involved in polyketide chain termination. ${ }^{19}$ By analogy with other modular PKS, the MlsA2 type I TE would catalyse cyclisation to form the 12-membered core macrolactone. The MlsB type I TE might catalyse transfer of the side-chain polyketide chain to coenzyme A, to provide the active donor for esterification of the core lactone, ${ }^{19}$ or alternatively it might itself catalyse the direct acyl transfer to the core lactone. The type I TE domains are essentially identical in sequence, except for a few additional amino acids at the C-terminus of MlsA2 type I TE. They differ however from conventional chain-terminating TE domains of modular PKS in the apparent absence of the expected His and Asp/Glu residues, which form part of the highlyconserved triad of amino acid residues (Ser-His-Asp/Glu) characteristic of these enzymes. Also, the sequence around the predicted Ser active site nucleophile (X-X-Ser-X-X) deviates significantly from the consensus sequence (Gly-X-Ser-X-Gly) for these enzymes. Homology modelling of the TE-I of MlsA2 (B. Popovic, A. Moore and P. F. L., unpublished data) has confirmed that the active site shows significant differences from the arrangement in authentic TE active sites. Either the Mls TE-I domains represent a new sub-class of $\alpha, \beta$-hydrolases, or they are inactive, and other enzymes catalyse the required steps in mycolactone assembly.

\subsection{Role of auxiliary enzymes in mycolactone biosynthesis}

The mycolactone gene cluster contains the gene cyp140A7 (MUP_053) encoding a cytochrome P450 hydroxylase. The observed distribution of this enzyme in different mycolactoneproducing strains strongly suggests that it is responsible for the specific hydroxylation of mycolactone $\mathrm{C}$ at $\mathrm{C}-12^{\prime}$ to produce mycolactone A/B (Fig. 1), although this conversion has not yet been reproduced in vitro with purified enzyme. No other natural mycolactones undergo such hydroxylation in the side-chain, a modification that seems to correlate with increased cytotoxicity (section 4). When the cyp140A7 gene was expressed in the mycolactone F-producing strain M. marinum DL045, a novel mycolactone (mycolactone G) was produced (Fig. 1), whose structure reveals that hydroxylation had apparently occurred, not as expected on $\mathrm{C}-10^{\prime}$, but on the neighbouring methyl branch at $\mathrm{C}-8^{\prime}$, presumably because of subtle alterations in the geometry of the enzyme-substrate interaction. ${ }^{23}$

Another auxiliary enzyme encoded in the $\mathrm{mls}$ gene cluster is a discrete thioesterase (type II TE), an activity which is found associated with most modular PKS and which is thought to have a 'proof-reading' function. ${ }^{47}$ The mycolactone type II TE has been expressed in E. coli and shown to catalyse the hydrolysis of model thioester substrates as for authentic type II TE enzymes (A. Moore and P. F. L., unpublished data) but this does not rule out additional roles for this enzyme. Given that the type I TE domains of the mycolactone PKS are atypical, and possibly even inactive, it is interesting that phylogenetic analysis of the mycolactone type II TE shows that it has closest similarity not to 'proof-reading' type II TE enzymes, but rather to chain-terminating thioesterases of the MonCII family, involved in release of intermediates in polyether biosynthesis. ${ }^{48}$ Finally, the mycolactone gene cluster contains a gene encoding a ketosynthase-like (FabH-like) enzyme, MUP_045, which has been proposed to act as the acyltransferase that couples the activated polyunsaturated side-chain of mycolactone onto the core. ${ }^{19}$ This hypothesis remains to be tested by biochemical studies with purified enzyme and the appropriate substrates.

\section{Outlook}

Studies of mycolactones have been hampered by the slow growth of the host mycobacteria, the relatively small amounts of material produced and the high frequency of spontaneous lossof-function mutations in the $m l s$ genes. Altogether, these factors lead to considerable difficulties in producing sufficient quantities of material for structural studies or other research. There are no reports yet of transfer of pMUM001 or of the $\mathrm{mls}$ and mycolactone accessory genes, to permit mycolactone production in a more tractable heterologous host, and this remains a formidable challenge.

Meanwhile, although informative for the biology of M. ulcerans, the structural variation of natural mycolactones is 
presently too limited to allow detailed structure-function studies that might shed further light on the molecular mechanisms of mycolactone action. Structural variants with lower cytotoxicity or immunosuppressive activity might compete with mycolactone for binding to its molecular target, and thereby constitute valuable functional inhibitors of toxin production. Alternative strategies for generation of mycolactone variants are clearly needed, and in the absence of a system for heterologous expression of the genes which would open the way to engineered biosynthesis of new analogues, the most promising route to such analogues is offered by total chemical synthesis.

The first synthetic route to mycolactone A/B was reported in $2002,{ }^{11-13}$ making use of an earlier route to the side-chain proposed by others. ${ }^{49}$ More recently Kishi and colleagues have published a significantly more efficient synthetic route to this molecule, ${ }^{50}$ as well as syntheses of mycolactones $\mathrm{C}^{17}$ and $\mathrm{F}^{25}$ Several other groups have also recently reported their efforts to generate general and flexible routes to analogues of the mycolactone core ${ }^{51,52}$ and side-chain..$^{53,54}$ If successful, this work would allow direct testing of the proposed functions of the enzymes encoded in the $m l s$ locus, and also provide important tools for exploration of the enigmatic mechanism of action of mycolactone.

\section{Acknowledgements}

We gratefully acknowledge the financial support of the Wellcome Trust (H. H. and P. F. L.) and the National Health and Medical Research Council of Australia (T. S.).

\section{References}

1 D. H. Connor and H. F. Lunn, Int. J. Lepr., 1965, 33, supplement, p. 698.

2 H. F. Lunn, D. H. Connor, N. E. Wilks, G. R. Barnley, F. Kamunvi, J. K. Clancey and J. D. Bee, E. Afr. Med. J., 1965, 42, 275.

3 J. K. Read, C. M. Heggie, W. M. Meyers and D. H. Connor, Infect. Immun., 1974, 9, 1114.

4 M. Pimsler, T. A. Sponsler and W. M. Meyers, J. Infect. Dis., 1988, 157, 577.

5 K. M. George, L. P. Barker, D. M. Welty and P. L. C. Small, Infect. Immun., 1998, 66, 587.

6 A. A. Pahlevan, D. J. M. Wright, C. Andrews, K. M. George, P. L. C. Small and B. M. Foxwell, J. Immun., 1999, 163, 3928.

7 K. M. George, D. Chatterjee, G. Gunawardana, D. Welty, J. Hayman, R. Lee and P. L. C. Small, Science, 1999, 283, 854.

8 K. M. George, L. Pascopella, D. M. Welty and P. L. C. Small, Infect. Immun., 2000, 68, 877.

9 G. Gunawardana, D. Chatterjee, K. M. George, P. J. Brennan, D. Whittern and P. L. C. Small, J. Am. Chem. Soc., 1999, 121, 6092.

10 L. Marsollier, P. Brodin, M. Jackson, J. Kordulokova, P. Tafalmeyer, E. Carbonelle, J. Aubry, G. Milon, P. Legras, J. P. Saint Andre, C. Leroy, J. Cottin, M. L. J. Guillou, G. Reysset and S. T. Cole, PLoS Path., 2007, 3, e62.

11 A. B. Benowitz, S. Fidanze, P. L. C. Small and Y. Kishi, J. Am. Chem. Soc., 2001, 123, 5128.

12 S. Fidanze, S. F. Song, M. Szoslek-Pinaud, P. L. C. Small and Y. Kishi, J. Am. Chem. Soc., 2001, 123, 10117.

13 F. Song, S. Fidanze, A. B. Benowitz and Y. Kishi, Org. Lett., 2002, 4, 647.

14 L. D. Cadapan, R. L. Arslanian, J. R. Carney, S. M. Zavala, P. L. Small and P. Licari, FEMS Microbiol. Lett., 2001, 205, 385.

15 H. Hong, P. J. Gates, J. Staunton, T. Stinear, S. T. Cole, P. F. Leadlay and J. B. Spencer, Chem. Commun., 2003, 2822.

16 A. Mve-Obiang, R. E. Lee, F. Portaels and P. L. C. Small, Infect. Immun., 2003, 71, 774.
17 T. C. Judd, A. Bischoff, Y. Kishi, S. Adusumilli and P. L. Small, Org. Lett., 2004, 6, 4901.

18 H. Hong, J. B. Spencer, J. L. Porter, P. F. Leadlay and T. Stinear, ChemBioChem, 2005, 6, 643.

19 T. Stinear, A. Mve-Obiang, P. L. Small, W. Frigui, M. J. Pryor, R. Brosch, G. A. Jenkin, P. D. Johnson, J. K. Davies, R. E. Lee, S. Adasumilli, T. Garnier, S. F. Haydock, P. F. Leadlay and S. T. Cole, Proc. Natl. Acad. Sci. U. S. A., 2004, 101, 1345.

20 K. A. Trott, B. A. Stacy, B. D. Lifland, H. E. Diggs, R. M. Harland, M. K. Khokha, T. A. Grammer and J. M. Parker, Comp. Med., 2004, 54, 309 .

21 A. Mve-Obiang, R. E. Lee, E. S. Umstot, K. A. Trott, T. C. Grammer, J. M. Parker, B. S. Ranger, R. Grainger, E. A. Mahrous and P. L. Small, Infect. Immun., 2005, 73, 3307.

22 H. Hong, T. Stinear, P. Skelton, J. B. Spencer and P. F. Leadlay, Chem. Commun., 2005, 34, 4306.

23 H. Hong, T. Stinear, J. L. Porter, C. Demangel and P. F. Leadlay, ChemBioChem, 2007, 8, 2043.

24 B. S. Ranger, E. A. Mahrous, L. Mosi, S. Adusumilli, R. E. Lee, A. Colorni, M. Rhodes and P. L. Small, Infect. Immun., 2006, 74, 6037.

25 H. J. Kim and Y. Kishi, J. Am. Chem. Soc., 2008, 130, 1842.

26 D. S. Snyder and P. L. C. Small, Microbial Pathogenesis, 2003, 34, 91.

27 E. Coutanceau, J. Decalf, A. Martino, A. Babon, N. Winter, S. T. Cole, M. L. Albert and C. Demangel, J. Exp. Med., 2007, 204, 1395.

28 S. Adusumilli, A. Mve-Obiang, T. Sparer, W. Meyers, J. Hayman and P. L. C. Small, Cell. Microbiol., 2005, 7, 1295.

29 E. Coutanceau, L. Marsollier, R. Brosch, E. Perret, P. Goossens, M. Tanguy, S. T. Cole, P. L. C. Small and C. Demangel, Cell. Microbiol., 2005, 7, 1187.

30 E. Torrado, S. Adusumilli, A. G. Fraga, P. L. Small, A. G. Castro and J. Pedrosa, Infect. Immun., 2007, 75, 3979.

31 J. Guarner, J. Bartlett, E. A. S. Whitney, P. L. Raghunathan, Y. Stienstra, K. Asamoa, S. Etuaful, E. Klutse, E. Quarshie, T. S. van der Werf, W. T. A. van der Graaf, C. H. King and D. A. Ashford, Emerging Infect. Dis., 2003, 9, 651.

32 T. M. Gooding, P. D. R. Johnson, D. E. Campbell, J. A. Hayman, E. L. Hartland, A. S. Kemp and R. M. Robins-Browne, Infect. Immun., 2001, 69, 1704-1707.

33 T. M. Gooding, P. D. R. Johnson, M. Smith, A. S. Kemp and R. M. Robins-Browne, Infect. Immun., 2002, 70, 5562.

34 T. M. Gooding, A. S. Kemp, R. M. Robins-Browne, M. Smith and P. D. R. Johnson, Clin. Infect. Dis., 2003, 36, 1076.

35 R. Phillips, C. Horsfield, S. Kuijper, S. F. Sarfo, J. Obeng-Baah, S. Etuaful, B. Nyamekye, P. Awuah, K. M. Nyarko, F. OseiSarpong, S. Lucas, A. H. Kolk and M. Wansbrough-Jones, Clin. Vaccine Immunol., 2006, 13, 253.

36 G. Prevot, E. Bourreau, H. Pascalis, R. Pradinaud, A. Tanghe, K. Huygen and P. Launois, Infect. Immun., 2004, 72, 958.

37 B. D. Westenbrink, Y. Stienstra, M. G. Huitema, W. A. Thompson, E. O. Klutse, E. O. Ampadu, H. M. Boezen, P. C. Limburg and T. S. van der Werf, Clin. Diagn. Lab. Immunol., 2005, 12, 125.

38 D. Yeboah-Manu, E. Peduzzi, E. Mensah-Quainoo, A. Asante-Poku, D. Ofori-Adjei, G. Pluschke and C. A. Daubenberger, J. Leukocyte Biol., 2006, 79, 1150.

39 K. J. Weissman and P. F. Leadlay, Nat. Rev. Microbiol., 2005, 3, 925.

40 M. J. Yip, J. L. Porter, J. A. M. Fyfe, C. J. Lavender, F. Portaels, M. Rhodes, H. Kator, A. Colorni, G. A. Jenkin and T. Stinear, J. Bacteriol., 2007, 189, 2021.

41 M. W. Rhodes, H. Kator, A. McNabb, C. Deshayes, J. M. Reyrat, B. A. Brown-Elliott, R. Wallace, Jr., K. A. Trott, J. M. Parker, B. Lifland, G. Osterhout, I. Kaattari, K. Reece, W. Vogelbein and C. A. Ottinger, Int. J. Syst. Evol. Microbiol., 2005, 55, 1139.

42 M. Ucko and A. Colorni, J. Clin. Microbiol., 2005, 43, 892.

43 J. F. Aparicio, I. Molnár, T. Schwecke, A. König, S. F. Haydock, L. E. Khaw, J. Staunton and P. F. Leadlay, Gene, 1996, 169, 9.

44 T. P. Stinear, H. Hong, W. Frigui, M. J. Pryor, R. Brosch, T. Garnier, P. F. Leadlay and S. T. Cole, J. Bacteriol., 2005, 187, 1668.

45 S. Bali and K. J. Weissman, ChemBioChem, 2007, 8, 2043.

46 P. Caffrey, ChemBioChem, 2003, 4, 654.

47 M. L. Heathcote, J. Staunton and P. F. Leadlay, Chem. Biol., 2001, 8, 207.

48 B. M. Harvey, H. Hong, M. A. Jones, Z. A. Hughes-Thomas, R. M. Goss, M. L. Heathcote, V. M. Bolanos-Garcia, W. Kroutil, J. Staunton and P. F. Leadlay, ChemBioChem, 2006, 7, 1435. 
49 M. K. Gurjar and J. Cherian, Heterocycles, 2001, 55, 1095.

50 F. Song, S. Fidanze, A. B. Benowitz and Y. Kishi, Tetrahedron, 2007, 63, 5739 .

51 M. D. Alexander, S. D. Fontaine, J. J. La Clair, A. G. Dipasquale, A. L. Rheingold and M. D. Burkart, Chem. Commun., 2006, 4602.
52 F. Feyen, A. Jantsch and K. Altmann, Synlett, 2007, 415-418.

53 R. P. Van Summeren, B. L. Feringa and A. J. Minnaard, Org. Biomol. Chem., 2005, 3, 2524

54 N. Yin, G. Wang, M. Qian and E. Negishi, Angew. Chem., Int. Ed., 2006, 45, 2916. 